TẠP CHÍ KHOA HỌC ĐẠI HỌC TÂN TRÀO
ISSN: 2354 - 1431

\title{
ÚNG DỤNG MÔ HÌNH LỚP HỌC ĐẢO NGƯợC TRONG DẠY HỌC NGOẠI NGŨ̃ NGHIÊN CỨU TÌNH HUỐNG GIẢNG DẠY TIẾNG ANH PHÁP LÝ TẠI TRƯờNG ĐẠI HỌC LUẬT HÀ NỘI
}

\author{
Vũ Văn Tuấn ${ }^{1 *}$ \\ ${ }^{1}$ Đại học Luật Hà Nội \\ *Email: vuvantuanphd@gmail.com
}

\section{Thông tin bài viết}

Ngày nhận bài:

28/6/2020

Ngày duyệt đăng:

20/9/2020

Từ khóa:

mô hình lớp học đảo ngươc, lóp thưc nghiệm, lóp truyền thống, tiếng Anh pháp lý

\section{Tóm tắt}

Mô hình lớp học đảo ngược có thể được xem là một trong những mô hình lớp học đáp ứng được nhu cầu của người dạy và người học trong thời đại công nghệ 4.0 hiện nay. Bài nghiên cứu này sử dụng mô hình bán thực nghiệm đối với 60 sinh viên đang theo học tiếng Anh chuyên ngành pháp lý, chia làm nhóm quan sát và nhóm thực nghiệm. Kết quả đã chỉ ra rằng sinh viên trải nghiệm trong lớp học đảo ngược đạt được hiệu quả ưu việt hơn so với lớp truyền thống. Trên cơ sở đó đề xuất ứng dụng mô hình lớp học đảo ngược trong dạy học tiếng Anh pháp lý tại Trường Đại học Luật Hà Nội.

\section{1. Đặt vấn đề}

Công nghệ thông tin đóng một vai trò ngày càng quan trọng trong giáo dục và cải tiến phương pháp giảng dạy hiện đại trên cơ sở phương pháp giảng dạy truyền thống. Một trong những sự lựa chọn này là mô hình lớp học đảo ngược (flipped classroom). Mô hình này giúp người học nghiên cứu lý thuyết thông qua việc xem các bài giảng đã được cập nhật trên hệ thống trực tuyến trước khi đến lớp. Điều này sẽ tiết kiệm thời gian học thực trên lớp cho các hoạt động thảo luận chuyên sâu, áp dụng lý thuyết tự học trên hệ thống vào việc giải quyết các vấn đề hoặc thực hành nhóm. Tăng cường sự tương tác giữa người học với người dạy.

Tiếng Anh chuyên ngành pháp lý là một đặc thù chuyên biệt góp phần tạo nên thương hiệu trường Đại học Luật Hà Nội. Vậy làm thế nào để người học có thể phát huy được khả năng của mình trong việc nâng cao năng lực ngôn ngữ trong lĩnh vực chuyên ngành là một trong những mong muốn của nhà quản lý, người dạy, và người học. Không giống như tiếng Anh giao tiếp, người học tiếng Anh chuyên ngành - đặc biệt là chuyên ngành pháp lý phải kết hợp tốt năng lực tiếng Anh cơ bản và kiến thức chuyên ngành mới có thể hiểu và sử dụng được tiếng Anh chuyên ngành. Thời lượng dành cho giảng dạy tiếng Anh chuyên ngành pháp lý còn hạn chế bên cạnh đó khối kiến thức tiếng Anh chuyên ngành lại đòi hỏi phải có thời gian dài cho người học và người dạy phát huy được hiệu quả của quá trình đào tạo. Chính vì vậy việc áp dụng mô hình lớp học đảo ngược có thể được xem như là một giải pháp hiệu quả giúp cho không chỉ người dạy có thể hỗ trợ tối đa cho người học, mà giúp người học vận dụng tốt lý thuyết tự học trước khi tham gia vào bài học thực hành trên lớp học. Bài nghiên cứu này giải đáp cho những vấn đề sau; 
1. Thái độ của người học với mô hình lớp học đảo ngược như thế nào?

2. Việc áp dụng mô hình lớp học đảo ngược tác động như thế nào đối với kết quả học tập của sinh viên?

\section{Cơ sở lý luận}

\subsection{Khái niệm về mô hình lớp học đảo ngược}

Có rất nhiều các khái niệm khác nhau về mô hình lớp học đảo ngược. Theo Bergmann \& Sams (2012) [1] định nghĩa mô hình lớp học đảo ngược là một mô hình sư phạm mà ở đó bài giảng và các bài tập về nhà trong khoá học được hoán đổi cho nhau. Bishop \& Verleger (2013) [2] nhận định rằng mô hình lớp học đảo ngược là một phương pháp học tập lấy người học làm trung tâm; gồm 2 phần là các hoạt động tương tác trong giờ học và hoạt động dạy của cá nhân chủ yếu dựa vào các bài giảng qua máy tính. Mull (2012) [3] miêu tả mô hình lớp học đảo ngược là một mô hình lớp học mà người học phải tự chuẩn bị các bài học bằng việc xem các video bài giảng, nghe các tệp âm thanh và đọc các tài liệu liên quan đến buổi học. Milman (2012) [4] cho rằng mô hình lớp học đảo ngược là một đường hướng nhằm đến tính hiệu quả của các bài học bằng việc truyền tải kiến thức tới người học thông qua các video bài giảng, các tệp âm thanh cũng như là các buổi thảo luận, làm việc nhóm và việc ứng dụng các thiết bị công nghệ trong suốt khoá học. Toto \& Nguyen (2009) [5] diễn tả mô hình lớp học đảo ngược là một phương pháp giúp tăng các hoạt động học tập tích cực và tạo cơ hội cho người học vận dụng kiến thức của mình trong lớp học dưới sự hướng dẫn của người dạy. Lý do các khái niệm về mô hình lớp học đảo ngược khác nhau là bởi vì các nhà giáo dục ở các quốc gia khác nhau sử dụng mô hình lớp học đảo ngược kết hợp với nhiều phương pháp khác nhau.

\section{2. Ư nhược điểm của mô hình lớp học đảo ngực}

\subsubsection{Uu điểm}

Theo Fulton (2012) [6] mô hình lớp học đảo ngược làm tăng thời gian tương tác trên lớp học. Thông qua các bài giảng được ghi hình và chuyển lên hệ thống mạng, người dạy dùng thời gian trên lớp để tương tác các hoạt động thực hành liên quan đến nội dung bài học với người học thay vì giảng bài. Còn Goodwin \& Miller (2013) [7] thì khẳng định rằng mô hình lớp học đảo ngược giúp người dạy có nhiều thời gian hơn để đáp ứng nhu cầu học tập và nhu cầu cảm xúc của người học. Bergmann \& Wadell (2012) [8] chỉ ra rằng trong mô hình lớp học đảo ngược, người học có thể tìm thấy cơ hội thảo luận với giáo viên của mình, cơ hội này thường khó gặp trong mô hình lớp học truyền thống.

Milman (2012) [4] nhận định rằng lợi ích quan trọng nhất của mô hình lớp học đảo ngược là hỗ trợ hoạt động nhóm trong lớp học. Những ưu điểm mà Fulton (2012) [6] nhấn mạnh là sinh viên có thể tiếp cận các video bài giảng bất cứu khi nào, bất cứ ở đâu học muốn và điều này giúp sinh viên học tập phù hợp với tốc độ học tập của bản thân. Kellinger (2012) [9] đã chứng minh rằng người học trong mô hình lớp học đảo ngược được khuyến khích tư duy cả trong và ngoài lớp học. Còn Herreid \& Schiller (2013) [10] khẳng định rằng mô hình lớp học đảo ngược giúp người học có nhiều thời gian hơn để tiến hành các nghiên cứu sáng tạo.

\subsubsection{Nhược điểm}

Mặc dù mô hình lớp học đảo ngược có nhiều điểm tích cực, song nó cũng có một số hạn chế nhất định. Một trong những vấn đề là sinh viên thụ động có thể không chuẩn bị bài trước khi đến lớp. Bên cạnh đó, các giờ giảng ghi hình cần được chuẩn bị một cách cẩn thận cho sinh viên của từng khoá học cụ thể. Theo Herreid \& Schiller (2013) [10] thì việc chuẩn bị các video bài giảng có chất lượng tốt thì không phải là dễ và cũng mất rất nhiều thời gian. Ngoài ra, các mô hình bài giảng được thiết kế để sử dụng trong mô hình lớp học đảo ngược còn hạn chế (Springen, 2013) [11].

Kordyban \& Kinash (2013) [12] chỉ ra khó khăn của người dạy khi phải đảm bảo người học có trách nhiệm trong việc chuẩn bị bài bên ngoài lớp học. Trong khi đó Bristol (2014) nhận định rằng giảng viên gặp rất nhiều khó khăn trong trường hợp người học đến lớp mà không chuẩn bị gì. Ngoài ra, việc người học thiếu các thiết bị công nghệ như điện thoại thông minh, máy tính bảng, máy tính cá nhân hoặc gặp những vấn đề về đường truyền Internet cũng được xem là những cản trở trong việc áp dụng mô hình lớp học đảo ngược (Kordyban \& Kinash, 2013) [12].

Hạn chế lớn nhất đối với người dạy không phải là việc chuẩn bị bài giảng hay là gửi các bài giảng đã ghi hình lên hệ thống, mà là việc chuẩn bị các hoạt động trong lớp học và tích hợp các hoạt động đó trong mô hình lớp học mô hình lớp học đảo ngược. Do đó, Lafee (2013) [13] kết luận rằng phương pháp này tăng cường trách nhiệm của người dạy đối với bài học thay vì coi đó như là một hoạt động đơn giản hơn so với cách giảng dạy truyền thống. 


\section{Phương pháp nghiên cứu}

\subsection{Thiết kế nghiên cứu}

Nghiên cứu này đánh giá sự cảm nhận của người học và tính hiệu quả của mô hình lớp học đảo ngược trong việc giảng dạy tiếng Anh pháp lý. Sử dụng mô hình bán thực nghiệm, tác giả đã sử dụng kết quả học tập của hai nhóm sinh viên tham gia vào nghiên cứu. Thông qua phiếu khảo sát và kết quả của bài thi đầu vào, đầu ra giữa nhóm thực nghiệm và quan sát, người nghiên cứu đã thu được những kết quả để trả lời câu hỏi nghiên cứu đã đặt ra. Từ đó, tác giả đề xuất ứng dụng mô hình lớp học phù hợp trong việc dạy tiếng Anh chuyên ngành pháp lý tại trường Đại học Luật Hà Nội trong thời đại công nghệ 4.0.

\subsection{Phương pháp nghiên cúu}

Phương pháp miêu tả định lượng so sánh được sử dụng dựa trên mô hình bán thực nghiệm với hai nhóm sinh viên tham gia đó là nhóm trải nghiệm và nhóm quan sát để tìm ra sự cảm nhận của người học đối với mô hình lớp học đảo ngược. Sử dụng bài kiểm tra đầu vào và đầu ra để làm căn cứ so sánh hiệu quả của mô hình lớp học đảo ngược so với mô hình lớp học truyền thống.

\section{3. Đối tự̆ng nghiên cứu}

Tác giả lựa chọn 60 sinh viên đang theo học tại 2 lớp tiếng Anh pháp lý của ngành luật Thương mại quốc tế học kỳ 2 năm học 2019-2020. Để thuận tiện cho việc quan sát và xử lý dữ liệu, tác giả gán lớp $\mathrm{A}$ (bao gồm 30 sinh viên) là lớp học theo mô hình đảo ngược, lớp $\mathrm{B}$ (bao gồm 30 sinh viên) là lớp theo học theo phương pháp truyền thống. Với đối tượng lớp $\mathrm{A}$ (flipped classroom), sinh viên kết hợp việc học trên lớp và truy cập vào hệ thống LMS (hệ thống học trực tuyến - Learning Management System), trong khi đó lớp $\mathrm{B}$ (lớp theo mô hình truyền thống) vẫn học theo phương pháp thông thường đó theo tiến độ chương trình đề cương môn học đề ra học lý thuyết và thực hành trên lớp với khung thời gian xác định.

\subsection{Công cụ nghiên cúu}

Dựa vào bảng khảo sát để đánh giá cảm nhận hiệu quả của mô hình lớp học đảo ngược. Nội dung bảng khảo sát gồm 2 phần, cụ thể phần thứ nhất điều tra về thông tin cá nhân người học bao gồm giới tính, nơi sinh sống và thiết bị công nghệ được sử dụng chủ yếu trong hoạt động học. Phần 2 gồm 14 câu hỏi do người nghiên cứu thiết kế dựa vào quan điểm thiết kế câu hỏi thái độ và cảm nhận (Donyei, 2010). Người học đánh giá theo 5 mức; (1) rất không đồng ý, (2) không đồng ý, (3) không xác định, (4) đồng ý, (5) rất đồng ý. 14 câu hỏi khảo sát tập trung vào 3 nội dung chính. Để tăng sự tin cậy đối với bảng khảo sát, tác giả đã trộn các câu hỏi lẫn lộn trong bảng khảo sát. Cụ thể, nhóm câu hỏi về cảm nhận của người học (gồm câu hỏi: 1, 3, 4, 7, 11), hoạt động của người học (gồm câu hỏi: $5,6,8,13$ ), thái độ của người học (gồm câu: 2, 9, 12, 14). Ban đầu tác giả xây dựng 25 câu hỏi thô, sau đó nhóm 25 câu hỏi được chuyển tới 2 chuyên gia giáo dục có kinh nghiệm để hỏi ý kiến thẩm định và phản biện. Tiếp đến, để đánh giá chính xác và tin cậy, bảng câu hỏi được sử dụng thử nghiệm đối với 10 sinh viên. Kết quả của thử nghiệm được đưa vào xử lý dữ liệu để kiểm định độ tin cậy thang đo Cronbach's Alpha. Tác giả đã loại bỏ 11 câu hỏi trong bảng câu hỏi thô vì không đáp ứng được tiêu chí của thang đo lường. Còn lại 14 câu hỏi trong khoảng $0.8-0.9$ được giữ lại để sử dụng trong bảng hỏi cuối cùng. Bảng câu hỏi khảo sát được chuyển tới 30 sinh viên lớp tiếng Anh pháp lý của ngành luật Thương mại quốc tế của trường Đại học Luật Hà Nội.

Phương pháp so sánh tương quan giữa hai nhóm dựa trên kết quả thi đầu vào thi đầu ra được áp dụng cho hai nhóm $\mathrm{A}$ và $\mathrm{B}$ nhằm đối chiếu và so sánh hai kết quả để thấy được sự khác biệt khi áp dụng mô hình lớp học đảo ngược giữa hai nhóm sinh viên. Sử dụng kết quả đầu ra của nhóm sinh viên $\mathrm{A}$ để kiểm định lại tính hiệu quả khi áp dụng mô hình lớp học đảo ngược.

\subsection{Thu thập và xử lý dĩ liệu}

Sau khi đạt được sự đồng thuận và tình nguyện của hai nhóm lớp học $\mathrm{A}$ và $\mathrm{B}$, tác giả sử dụng bài kiểm tra đầu vào áp dụng với 60 sinh viên. Đối với nhóm sinh viên trải nghiệm, sinh viên được giải thích kỹ về cách tham gia trong quá trình thử nghiệm học kỳ 2 năm học 2019-2020. Sau khi kết thúc học kỳ 2 , bài thi đầu ra được áp dụng với cả hai nhóm sinh viên. Ngoài ra, nhóm 30 sinh viên trải nghiệm sẽ trả lời thêm phần phiếu điều tra khảo sát cho mục đích nghiên cứu.

Sử dụng phần mềm IBM SPSS để xử lý dữ liệu mã hoá từ kết quả bài kiểm tra đầu vào và đầu ra, kết quả thu được từ phiếu khảo sát đối với nhóm sinh viên A. Sử dụng miêu tả tần xuất (descriptive frequency) để xử lý phần thu thập thông tin của đối tượng khảo sát. Tính trung vị (mean) để tìm ra quan điểm chung của sinh viên về 14 câu hỏi trong bảng khảo sát, được phân chia theo thang tỷ lệ Likert (1.0- 
1.79) rất không hài lòng, (1.8-2.59) không hài lòng, (2.6-3.39) không rõ ràng quan điểm, (3.4-4.19) hài lòng, (4.2-5.0) rất hài lòng. So sánh cặp (PairedSample $\mathrm{T}$ Test) được sử dụng để so sánh mức trung bình chung giữa điểm đầu vào và đầu ra để đánh giá sự hiệu quả khi áp dụng mô hình lớp học đảo ngược và mô hình lớp học truyền thống. Ngoài ra, hệ số tương quan Pearson được sử dụng để so sánh kết quả đầu ra của sinh viên thực nghiệm với việc áp dụng mô hình lớp học đảo ngược.

\section{Kết quả và bàn luận}

\section{1. Đặc điểm của đối tự̂ng tham gia khảo sát}

Khi khảo sát 30 sinh viên tham gia quá trình thực nghiệm, kết quả thu được 11 sinh viên nam chiếm $36.7 \%$, và 19 sinh viên nữ tương đương với $63.3 \%$. Hầu hết sinh viên đến từ khu vực nông thôn 16 sinh viên chiếm $55.3 \%$, số lượng sinh viên tham gia đến từ thành phố và các tỉnh miền núi tương đương nhau chiếm 7 sinh viên, tương đương $23.3 \%$. Khi được hỏi về phương tiện học tập, $2 / 3$ sinh viên sử dụng máy tính trong học tập (66.7\%), ngược lại 1/3 hoặc 33.3\% sinh viên sử dụng điện thoại thông minh để thay thế máy tính trong học tập.

\subsection{Kết quả đánh giá trải nghiệm của người học trong lớp học đảo ngực}

\subsubsection{Cảm nhận của người học.}

Bảng 1 miêu tả cảm nhận của sinh viên khi tham gia vào mô hình lớp học đảo ngược. Nhìn chung sinh viên cảm thấy hài lòng khi tham gia mô hình lớp học đảo ngược này. So với mô hình lớp học truyền thống, sinh viên tham gia mô hình lớp học đảo ngược đánh giá lớp học đảo ngược là một cách học tốt nhất $(M=$ $3.67, S D=.844$ ), sinh viên hài lòng khi tham gia vào lớp học này, và tỷ lệ lựa chọn sự hài lòng không khác nhau là mấy thông qua biểu hiện của mức độ lệch chuẩn $(S D=.844)$ dưới 1.0. Theo sau kết quả trên, sinh viên cho rằng thời gian trải nghiệm lớp học đảo ngược là rất đáng giá $(M=3.57, \mathrm{SD} .858)$. Sinh viên cũng cho rằng họ đã hình thành động cơ học tập tốt hơn trong lớp học đảo ngược $(M=3.60, S D=.9 .32)$, người học cũng đánh giá rằng hiệu quả và kết quả của lớp học đảo ngược tốt hơn so với lớp truyền thống $(M=3.53, S D=.900)$. Ngoài ra sinh viên cho rằng họ cảm nhận được sự trải nghiệm vui vẻ trong lớp học đảo ngược $(M=3.40, S D=.968)$. Tuy vậy, sinh viên vẫn cần có sự can thiệp của giảng viên trong việc hướng dẫn về các chủ đề của bài học. Dựa vào số liệu phân tích, với quan điểm này vẫn chưa được sinh viên xác định rõ ràng $(M=3.33)$. Xét về tổng thể sinh viên cảm nhận sự hài lòng khi tham gia vào mô hình lớp học đảo ngược (3.52).

Bảng 1. Cảm nhận của ngườ học trong lớp học đảo ngược

\begin{tabular}{l|l|c|c|c}
\hline \multicolumn{1}{|c|}{ Nội dung } & N & M & SD \\
\hline 1. & Lớp học đảo ngược là cách học tốt nhất & 30 & 3.67 & .844 \\
\hline 3. & Tôi nghĩ rằng lớp học đảo ngược thì hiệu quả hơn và kết quả tốt hơn. & 30 & 3.53 & .900 \\
\hline 4. & Tôi cảm thấy có động cơ học tập hơn trong lớp học đảo ngược & 30 & 3.60 & .932 \\
\hline 7. & Tôi nghĩ thời gian và nỗ lực dành cho lớp học đảo ngược này thật đáng giá. & 30 & 3.57 & .858 \\
\hline 10. & $\begin{array}{l}\text { Tôi nghĩ học trong lớp đảo ngược hướng dẫn tôi hiểu hơn về các chủ đề } \\
\text { của bài học. }\end{array}$ & 30 & 3.33 & .994 \\
\hline 11. & Tôi trải nghiệm sự vui vẻ trong lớp học đảo ngược & 30 & 3.40 & .968 \\
\hline & & Mức trung bình chung & 3.52 & \\
\hline
\end{tabular}

\subsubsection{Hoạt động của ngườ học}

Bảng 2 nêu ra các hoạt động của người học trong mô hình lớp học đảo ngược. Sinh viên cho rằng sinh viên năng động hơn trong lớp học đảo ngược $(M=$ $3.50, S D=.820$ ), học nhiều và học tốt hơn trong lớp học đảo ngược $(M=3.50, S D=.900)$. Người trải nghiệm cho rằng họ đã dành nhiều thời gian và nỗ lực hơn bình thường trong lớp học đảo ngược $(M=$ $3.47, S D=.860)$. Những chỉ số trong cột trung bình trung đã nói lên rằng sinh viên hài lòng khi tham gia vào lớp học trải nghiệm. Tuy vậy, sinh viên cũng chưa xác định rõ quan điểm có hay không khi tham gia và gắn kết bản thân mình hơn khi học tỏng lớp 
học đảo ngược $(M=3.30, S D=.952)$. Sự lựa chọn này không đồng nhất giữa các quan điểm khi độ lệch chuẩn biến dao động ở mức biến thiên cao hơn so với các chỉ số trong cùng nhóm câu hỏi $(S D=.952)$. Nhìn chung, sinh viên hài lòng với các hoạt động khi tham gia mô hình lớp học đảo ngược.

Bảng 2. Hoạt động của người học trong lớp học đảo ngược

\begin{tabular}{|c|c|c|c|c|}
\hline \multicolumn{2}{|r|}{ Nội dung } & $\mathbf{N}$ & $\mathbf{M}$ & SD \\
\hline 5. & $\begin{array}{l}\text { Tôi tham gia và gắn kết bản thân mình hơn khi học trong lớp học đảo } \\
\text { ngược. }\end{array}$ & 30 & 3.30 & .952 \\
\hline 6. & Tôi trở thành sinh viên năng động hơn trong lớp học đảo ngược. & 30 & 3.50 & .820 \\
\hline 8. & Tôi học nhiều và học tốt hơn trong lớp học đảo ngược & 30 & 3.50 & .900 \\
\hline \multirow[t]{2}{*}{13.} & $\begin{array}{l}\text { Tôi dành nhiều thời gian và nỗ lực hơn bình thường trong các hoạt động } \\
\text { học của lớp học đảo ngược }\end{array}$ & 30 & 3.47 & .860 \\
\hline & Weighted mean & & 3.44 & \\
\hline
\end{tabular}

\subsubsection{Thái độ của ngưòi học}

Bảng 3 xem xét đến thái độ của người học khi tham gia vào mô hình lớp học đảo ngược. Người học cảm nhận thấy hạnh phúc và thoả mãn khi được trải nghiệm mô hình lớp học đảo ngược $(M=3.73$, $S D=8.28$ ), số liệu này chỉ ra rằng người học có sự lựa chọn tương đối giống nhau khi nhìn vào độ lệch chuẩn của sự lựa chọn. Người học cũng thừa nhận rằng họ thích học theo phương pháp mô hình lớp học đảo ngược hơn so với lớp học truyền thống $(M=$ $3.67, S D=.959)$. Thông qua kết quả phiếu điều tra, người học nhận định rằng họ đã để tâm hơn vào các hoạt động cũng như chỉ dẫn trong lớp học đảo ngược, sự lựa chọn này có sự đồng nhất giữa người tham gia trả lời phiếu khảo sát $(M=3.53, S D=.776)$. Khi được hỏi về so sánh giữa lớp học truyền thống so với lớp học mô hình đảo ngược thì sinh viên nhất trí rằng lớp học theo mô hình đảo ngược hiệu quả hơn so với lớp truyền thống, mặc dù sự lựa chọn này không có tỷ lệ đồng đều giữa người tham gia $(M=3.47, S D=$ .973). Nhìn chung, sinh viên hài lòng khi tham gia vào lớp học mô hình đảo ngược này thông qua chỉ số bình quân gia quyền $(W M=3.60)$.

Bảng 3. Thái độ của người học trong lớp hoc đảo ngược

\begin{tabular}{|c|c|c|c|c|c|c|c|c|}
\hline \multicolumn{6}{|c|}{ Nội dung } & $\mathbf{N}$ & M & SD \\
\hline 2. & \multicolumn{5}{|c|}{ Tôi thích học theo phương pháp đảo ngược hơn. } & 30 & 3.67 & .959 \\
\hline 9. & \multicolumn{5}{|c|}{ Tôi thích lớp học đảo ngược hơn so với lớp học truyền thống. } & 30 & 3.47 & .973 \\
\hline 12. & \multicolumn{5}{|c|}{ Tôi đã để tâm hơn vào các hoạt động cũng như chỉ dẫn trong lớp học đảo ngược } & 30 & 3.53 & .776 \\
\hline \multirow[t]{2}{*}{14.} & \multicolumn{5}{|c|}{$\begin{array}{l}\text { Nói chung, tôi cảm thấy hạnh phúc và thoả mãn với sự trải nghiệm trong lớp } \\
\text { học đảo ngược. }\end{array}$} & 30 & 3.73 & .828 \\
\hline & \multicolumn{5}{|c|}{ Weighted mean } & & 3.60 & \\
\hline \multicolumn{9}{|c|}{$\begin{array}{l}\text { 4.3. Kết quả điểm số của sinh viên trong lớp học } \\
\text { mô hình đảo ngực } \\
\text { Bảng } 4 \text { thể hiện kết quả so sánh điểm bắt đầu } \\
\text { tham gia khoá học của hai nhóm sinh viên. Trong đó } \\
\text { nhóm A là sinh viên theo học mô hình lớp học đảo } \\
\text { Bảng 4. So sánh điểm đầu vào (Pre-test) giũa } 2 \text { lópp. }\end{array}$} \\
\hline \multicolumn{4}{|r|}{ Mean } & $\mathrm{N}$ & $\begin{array}{c}\text { Std. } \\
\text { Deviation }\end{array}$ & \multicolumn{3}{|c|}{ Std. Error Mean } \\
\hline \multirow{2}{*}{\multicolumn{2}{|c|}{$\begin{array}{l}\text { Bài kiểm } \\
\text { tra đầu vào }\end{array}$}} & A - lớp học đảo ngược & 6.97 & 30 & .65 & & & .120 \\
\hline & & $\mathrm{B}$ - lớp học truyền thống & 6.87 & 30 & .70 & & & .129 \\
\hline
\end{tabular}


Bảng 5 thể hiện sự khác biệt về chất lượng đào tạo giữa hai mô hình lớp học - Mô hình lớp học đảo ngược và mô hình lớp học truyền thống sau một học kỳ dựa trên bài kiểm tra đầu ra giống nhau được áp dụng với cả hai lớp. Kết quả phản ánh rõ rằng cả hai nhóm lớp đều có sự tiến bộ trong học tập nhưng sự ưu việt của ứng dụng mô hình lớp học đảo ngược $(M$

Bảng 5. So sánh điểm đầu ra (Post-test) giũa 2 lớp
= 8.58) đã thu lại được kết quả tốt hơn so với lớp học truyền thống $(M=7.02)$. So với kết quả ban đầu khoá học, cả hai lớp có xuất phát điểm giống nhau nhưng kết quả thu được sau thời gian thử nghiệm lại khác nhau rõ rệt. Mô hình lớp học đảo ngược đã mang lại hiệu quả hơn so với lớp học truyền thống.

\begin{tabular}{|c|c|c|c|c|c|}
\hline & & Mean & $\mathrm{N}$ & $\begin{array}{c}\text { Std. } \\
\text { Deviation }\end{array}$ & Std. Error Mean \\
\hline \multirow{2}{*}{$\begin{array}{l}\text { Bài kiểm } \\
\text { tra đầu ra }\end{array}$} & A - lớp học đảo ngược & 8.58 & 30 & .527 & .096 \\
\hline & $\mathrm{B}$ - lớp học truyền thống & 7.02 & 30 & .609 & .111 \\
\hline
\end{tabular}

Bảng 6 thể hiện mối tương quan giữa việc áp dụng mô hình đảo ngược so với kết quả đầu ra của nhóm trải nghiệm mô hình phương pháp đảo ngược, dựa trên giả thuyết rằng việc áp dụng mô hình lớp học đảo ngược này không có tác dụng đối với sinh

viên. Kết quả cho thấy rằng hệ số tương quan Pearson là -.172 nhỏ hơn mức xác định $1 \%$, bên cạnh đó hệ số quan sát Sig. (2-tailed) là .363 lớn hơn 0.05 . Như vậy, bác bỏ giả thuyết mô hình lớp học đảo ngược không có hiệu quả so sánh với lớp học truyền thống

Bảng 6. Mối liên hệ giữa việc áp dụng mô hình lớp học đảo ngược với kết quả đầu ra

Flipped classroom

A post-test

\begin{tabular}{|c|c|c|c|}
\hline \multirow[t]{3}{*}{ Lớp học đảo ngược } & Pearson Correlation & 1 & -.172 \\
\hline & Sig. (2-tailed) & & .363 \\
\hline & $\mathrm{N}$ & 30 & 30 \\
\hline \multirow{3}{*}{$\begin{array}{l}\text { Bài kiểm tra đầu ra } \\
\text { lớp học đảo ngược }\end{array}$} & Pearson Correlation & -.172 & 1 \\
\hline & Sig. (2-tailed) & .363 & \\
\hline & $\mathrm{N}$ & 30 & 30 \\
\hline
\end{tabular}

\section{Kết luận và khuyến nghị}

\subsection{Kết luận}

Qua việc phân tích số liệu đã chứng minh rằng người học có thái độ tích cực khi trải nghiệm mô hình lớp học đảo ngược trong lớp học tiếng Anh pháp lý. Kết quả của phiếu điều tra về sự cảm nhận của người học, hoạt động của người học và thái độ của người học trong bảng khảo sát đã chỉ ra rằng người học nhìn chung đã hài lòng với việc ứng dụng mô hình lớp học đảo ngược trong việc học ngôn ngữ nói chung và tiếng Anh chuyên ngành pháp lý nói riêng. Sinh viên tham gia lớp học trải nghiệm mô hình lớp học đảo ngược đã có sự chuyển biến về trạng thái tâm lý tích cực tham gia vào các hoạt động thực tiễn trên lớp. Giảng viên linh hoạt vận dụng các tài liệu thực hành liên quan đến bài học trên lớp với kiến thức lý thuyết đã được sinh viên học trước trên hệ thống. Các hoạt động lớp học cũng được tham gia sôi nổi bởi vì sinh viên ứng dụng kiến thức lý thuyết mình nắm vững trước khi đến với bài học, không còn trạng thái tâm lý thụ động khi giảng viên dành thời gian giải thích hay hướng dẫn như trong lớp học truyền thống đang diễn ra.
Kết quả sau khi áp dụng mô hình lớp học đảo ngược cũng chứng minh rằng tính hiệu quả của mô hình này vượt trội hơn so với lớp học truyền thống. Mặc dù có chung điểm xuất phát thể hiện qua bài thi đầu vào nhưng kết quả ở bài thi đầu ra có sự khác biệt đáng kể. Kết hợp với kết quả của phiếu điều tra khảo sát đối với sinh viên trải nghiệm mô hình lớp học đảo ngược, có thể thấy rằng mô hình lớp học đảo ngược đã mang lại hiệu quả tích cực với người học không những chỉ kiến thức trên lớp mà còn thay đổi trạng thái tâm lý khi tham gia học tập.

\section{Hạn chế của đề tài}

Mô hình lớp học đảo ngược này mới chỉ áp dụng hạn chế số lượng sinh viên ở một chuyên ngành, một môi trường hẹp. Cần nhân rộng thí điểm mô hình lớp học đảo ngược này ở nhiều ngành cũng như nhiều trường đại học khác nhau để kết luận liệu như mô hình lớp học đảo ngược này có mang lại hiệu quả tích cực đối với ngành giáo dục hay không khi nền công nghiệp 4.0 đang tác động đến mọi mặt của đời sống, và giáo dục không phải là ngoại lệ. 


\subsection{Khuyến nghị}

Đối với nhà trường: Cần nhân rộng mô hình lớp học đảo ngược vào giảng dạy các môn học tại trường Đại học Luật Hà Nội nói chung và các môn tiếng Anh chuyên ngành pháp lý nói riêng. Nhà trường cần đầu tư cơ sở vật chất chuyên biệt phục vụ cho việc biên soạn các bài giảng giáo án điện tử, nền tảng công nghệ thông tin, đặc biệt là hệ thống MLS tốt nhất để giảng viên và sinh viên khai thác hiệu quả nhất. Cần có cơ chế đặc thù khuyến khích giảng viên tham gia vào việc giảng dạy mô hình lớp học đảo ngược như cơ chế tài chính hoặc bồi dưỡng chuyên môn nghiệp vụ. Nhà trường cần có sự kết hợp chặt chẽ giữa các đơn vị để phối hợp tạo môi trường tốt nhất khi triển khai mô hình lớp học đảo ngược này.

Đối với người dạy: Giảng viên cần tích cực chủ động tham gia xây dựng hệ thống bài giảng tương tác với sinh viên thông qua giáo án điện tử và tài liệu cập nhật trên lớp. Khai thác tối đa sự tự chủ học tập của sinh viên, khuyến khích sinh viên tích cực tham gia các hoạt động trên lớp. Giảng viên cần có các hoạt động giảng dạy đa dạng và có các "kịch bản sư phạm" phù hợp đối với từng nhóm sinh viên khác nhau trong một lớp. Cần gắn kết chặt chẽ nền tảng MLS với hoạt động cập nhật bài giảng trên hệ thống lớp học. Ngoài ra, giảng viên không ngừng trao đổi cập nhật kiến thức công nghệ thông tin cũng như các hoạt động sư phạm chuyên môn nhằm tạo môi trường học tập hiệu quả đối với sinh viên trong lớp học mô hình đảo ngược.

Đối với người học: Phát huy vai trò chủ động tích cực khi tham gia vào mô hình lớp học đảo ngược. Tăng cường tính tự học, tự nghiên cứu, tự giác trong học tập. Chủ động trong học lý thuyết trên hệ thống MLS và tích cực tham gia các hoạt động trên lớp do giảng viên thiết kế biên soạn như làm việc theo cặp, theo nhóm thực hành. Sinh viên cần chủ động trao đổi với giảng viên về những khó khăn trở ngại khi tham gia vào mô hình lớp học đảo ngược.

\section{REFERENCES}

[1]. Bergmann, J., \& Sams, A. (2012). Flipping for mastery. Educational Leadership, 71(4), 24-29. http://www.ascd.org/publications/educationalleadership/dec13/vol71/num04/Flipping-forMastery.aspx

[2]. Bishop, J. L., \& Verleger, M. A. (2013). The Flipped Classroom: A Survey of the Research. $120^{\text {th }}$ ASEE Annual Conference \& Exposition. Atlanta: GA. $30,1-18$
[3]. Mull B. (2012). Flipped learning: A response to five common criticisms. https://novemberlearning.com/wpcontent/uploads/2012/10/flipped-learning-a-responseto-five-common-criticisms.pdf

[4]. Milman, N. (2012). The flipped classroom strategy: what is it and how can it be used? Distance Learning, 9(3), 85-87. https://go.gale.com/ps/anonymous?id=GALE\%7CA30 $5660562 \&$ sid $=$ googleScholar $\& v=2.1 \& \mathrm{it}=\mathrm{r} \&$ linkacces $\mathrm{s}=\mathrm{abs} \& \mathrm{issn}=15474712 \& \mathrm{p}=\mathrm{AONE} \& \mathrm{sw}=\mathrm{w}$

[5]. Toto, R., \& Nguyen, H. (2009). Flipping the work design in an industrial engineering course. ASEE/IEEE Frontiers in Education Conference. San Antonio, TX

[6]. Fulton, K. (2012). Upside down and inside out: Flip your classroom to improve student learning. Learning \& Leading with Technology, 39(8), 12-17. Retrieve from https://eric.ed.gov/?id=EJ982840

[7]. Goodwin, B. \& Miller, K. (2013). Evidence on Flipped Classrooms Is Still Coming In. Educational Leadership, 70(6), 78-80. Retrieved from http://www.ascd.org/publications/educationalleadership/mar13/vol70/num06/Evidence-on-FlippedClassrooms-Is-Still-Coming-In.aspx.

[8]. Bergmann, J. \& Waddell, D. (2012). To flip or not to flip? Learning and Leading with Technology, 39(8), 6-7.

[9]. Kellinger, J. J. (2012). The flipside: Concerns about the "New literacies" paths educators might take. The Educational Forum, 76(4), 524-536. https://doi.org/10.1080/00131725.2012.708102

[10]. Herreid, F. C. \& Schiller, A. N. (2013). Case Studies and the Flipped Classroom. Journal of College Science Teaching, 42(5), 62-66. https://www.aacu.org/sites/default/files/files/PKAL_re gional/CRWG-SPEE-REF-01.pdf

[11]. Springen, K. (2013). Flipping the Classroom: A revolutionary approach to learning presents some pros and cons. School Library Journal, 59(4)23. https://www.slj.com/?detailStory=flipping-theclassroom-a-revolutionary-approach-to-learningpresents-some-pros-and-cons

[12]. Kordyban, R., \& Kinash, S. (2013). No more flying on auto pilot: The flipped classroom. Education Technology Solutions, 56, 54-56. https://pure.bond.edu.au/ws/portalfiles/portal/2794968 2/No_more_flying_on_autopilot_The_flipped_classro om.pdf

[13]. Lafee, S. (2013). Flipped learning. The Education Digest, 13-18. https://espforuniversityprep.weebly.com/uploads/2/8/8 /6/28861675/flipped_learning_lafree_2013.pdf 
APPLICATION OF THE REVERSED CLASS MODEL IN FOREIGN

LANGUAGE TEACHING. RESEARCH ON LEGAL ENGLISH LANGUAGE TEACHING AT HA NOI UNIVERSITY OF LAW

Article info

Recieved:

05/7/2020

Accepted:

20/9/2020

Keywords:

reversed classroom,

experimental class,

traditional group, legal

English

\section{Abstract}

Reversed classroom is considered one of the most innovative pedagogical models which meet the need of teachers and learners in the age of Industry 4.0 now. This study employed quasi-experiment on 60 students studying legal English subject, and they were divided into observation groups and experimental groups. The results showed that the experimental group had a better learning outcome over the traditional class. On that basis, it is proposed to apply the reversed classroom model in teaching and learning legal English at Hanoi University of Law. 